\title{
Statyba
}

\section{A GENERALIZED SYSTEMOTECHNICAL EVALUATION AND DETERMINATION OF THE PRIORITIES IN BUILDING DESIGN DECISIONS}

\section{E. K. Zavadskas}

To cite this article: E. K. Zavadskas (1995) A GENERALIZED SYSTEMOTECHNICAL EVALUATION AND DETERMINATION OF THE PRIORITIES IN BUILDING DESIGN DECISIONS, Statyba, 1:4, 31-39, DOI: 10.1080/13921525.1995.10531531

To link to this article: https://doi.org/10.1080/13921525.1995.10531531

Published online: 26 Jul 2012.

Submit your article to this journal $\llbracket$

Џ Article views: 47 


\section{ОБОБЩЕННАЯ СИСТЕМОТЕХНИЧЕСКАЯ ОЦЕНКА И ОПРЕДЕЛЕНИЕ ПРЕЛПОЧТИТЕЛЬНОСТИ ПРОЕКТНЫХ РЕШЕНИЙ В СТРОИТЕЛЬСТВЕ}

\section{Э.К.Завадскас}

\section{1. Введение}

При прогнозировании технических и организационно-технологических решений необходим учет уровня, достигнутого строительной наукой, и передового отечественного и зарубежного опыта проектирования и строительства. Достигнутый уровень должны характеризовать утвержденные прогрессивные удельные показатели (УПУП).

Проектные решения должны быть направлены на достижение этих величин, по возможности на улучшение некоторых УПУП и осуществляться на основе информации об уровне разработок, выполняемых каждой конкретной проектной организацией.

Представляется необходимым создание информационной базы в виде банка данных по достигнутым технико-экономическим показателям (ТЭІ) и качественным характеристикам (KX) для оценки типовых проектов, типовых проектных решений (ТПР), типовых конструкций, деталей и узлов. При определении конкретных ТЭП и КХ заказчик должен иметь возможность воспользоваться информационными данными проектной фирмы об уже созданных типах интересующего его проекта. При этом нельзя лишать заказчика некоторой свободы выбора и возможности отдать предпочтение некоторым ТЭП и КХ. Эти предпочтения могут быть заданы заказчиком в виде коэффициентов весомости ТЭП и $\mathrm{KX}$.

При наличии банка данных, записанного на носители информации компьютера, методов системотехнической оценки (СТОПР) [1] и многоцелевой селектоновации [2,3] вариантов на основе комплекса полидименсиональных ТЭП и $\mathrm{KX}$ у заказчика есть возможность обоснованно выбрать наиболее прогрессивный базовый проектный вариант и базовые значения ТЭП и КХ, которые и вносятся в техническое задание на проектирование. Таким образом, заказчик получает возможность прогнозировать ожидаемый результат проект здания или сооружения. Заказчик становится активным участником процесса принятия решения по выбору варианта проектного решения.

Для сравнения ранее разработанных вариантов проектных решений и определения их 
предпочтительности предлагается специально разработанная методика, алгоритмы и программы для персональных компьютеров. Специалисты проектных фирм в присутствии заказчика могут в диалоговом режиме выбрать базовый вариант проектного решения и согласовать базовые ТЭП и КХ, которые должны быть не хуже УПУП.

\section{2. Определение базовых технико-экономических показателей и качественных}

\section{характеристик}

Определение базовых ТЭП и КX осушествляется на основании этих параметров принятого в качестве базового проекта, выбор которого в свою очередь осуществляется на основании системотехнической оценки вариантов решений (СТОПР) из определенного множества ранее разработанных проектов аналогичного назначения.

Предлагаемый метод определения наиболее предпочтительного базового варианта проектного решения основан на формировании обобщенного критерия СТОПР (критерия полезности проекта $-\mathrm{K}_{\text {би }}$ ) на основе отклонений вариантов решений от так называемого "идеального проектного варианта", составленного из лучших значений полидименсиональных частных критериев оптимальности (ЧКО) - ТЭП и КХ сравниваемых вариантов решений. Близкая по смыслу математическая постановка определения преференций (предпочтений) на основе близости $\mathbf{x}$ идеальной точке рассмотрена в работе [4]. В рсзультате переработки и локальной интерпретации названного метода [5] получен эффективный способ определения обобшенного критерия СТОПР $\mathrm{K}_{\text {Бит }}$ многократно использованный автором при решении практических задач обоснования и выбора проектных решений $[6,7,8]$.

Все показатели эффективности (ЧКО) должны быть кардинальными, имеющиеся же ординальные (качественные ЧКО) показатели должны быть квантифицированы. Кроме того, должны быть определены или приняты равноценными коэффициенты весомости показателей эффективности. Методы определения коэффициентов весомости подробно изложены в работах $[1,5,8]$.

Решение задачи выбора базового варианта, определения базовых показателей и построения ряда предпочтительности всех разработанных ранее проектных решений осушествляется в несколько этапов. Приведем блок-схему разработанного алгоритма и дадим пояснение отдельных этапов.

Этап 1. Подготовка исходной информации по сравниваемым вариантам проектных решений. Представляется наиболее удобным и наглядным вводить значения полидименсиональных (разноразмерных) ЧКО (ТЭП и КХ) каждого проектного решения в память компьютера в виде матрицы (табл. 1). 


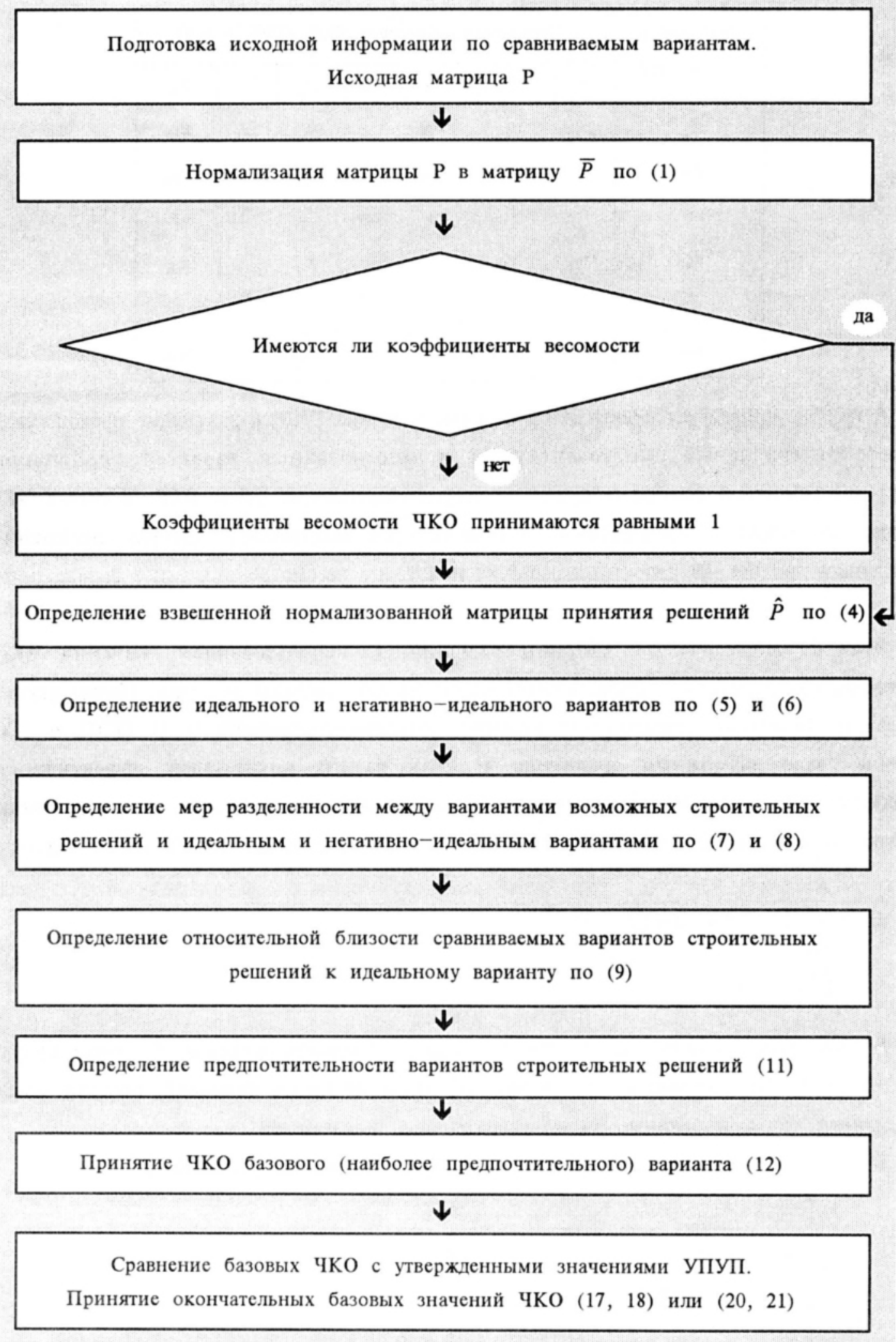

Схема определения предпочтительности сравниваемых строительных решений и бәзови значений тКО 
Частные критерии оптимальности проектных решенин

\begin{tabular}{|c|c|c|c|c|c|c|c|}
\hline \multirow{2}{*}{$\begin{array}{c}\text { Вариант ТР } \\
\text { или ОТР }\end{array}$} & \multicolumn{7}{|c|}{ Полидименсиональные ЧКО (ТЭП и $\mathrm{KX)}$} \\
\cline { 2 - 8 } & $x_{1}$ & $x_{2}$ & $x_{3}$ & $\cdots$ & $x_{j}$ & $\cdots$ & $x_{n}$ \\
\hline$B_{1}$ & $x_{11}$ & $x_{12}$ & $x_{13}$ & $\ldots$ & $x_{1 j}$ & $\ldots$ & $x_{1 n}$ \\
$B_{2}$ & $x_{21}$ & $x_{22}$ & $x_{23}$ & $\ldots$ & $x_{2 j}$ & $\ldots$ & $x_{2 n}$ \\
$\vdots$ & $\vdots$ & $\vdots$ & $\vdots$ & $\vdots::$ & $\vdots$ & $\vdots: \vdots$ & $\vdots$ \\
$B_{i}$ & $x_{i 1}$ & $x_{i 2}$ & $x_{i 3}$ & $\ldots$ & $x_{i j}$ & $\ldots$ & $x_{i n}$ \\
$\vdots$ & $\vdots$ & $\vdots$ & $\vdots$ & $:::$ & $\vdots$ & $\vdots: \vdots$ & $\vdots$ \\
$B_{m}$ & $x_{m 1}$ & $x_{m 2}$ & $x_{m 3}$ & $\ldots$ & $x_{m j}$ & $\ldots$ & $x_{m n}$ \\
\hline
\end{tabular}

В таблице приводятся полидименсиональные значения ЧКО, отражающие многоцелевой, многокритериальный, многоаспектный и многоэтапный характер обобщенной системотехнической оценки и принятия ресурсосберегающих ТР и ОТР. В состав ЧКО входят показатели, перечисляемые в номенклатуре показателей, которые необходимо учитывать при оценке проектных решений и которые рассмотрены в ранее выполненных работах автора $[1,5,8]$.

Этап 2. Нормализация матрицы решений. С использованием значений ЧКО, приведенных в таблице, осушествляется нормализация матрицы решений. Целью этапа 2 является получение безразмерных величин полидименсиональных ЧКО (ТЭП и КХ), чтобы стало возможным сравнение величин разных показателей эффективности принимаемых решений. Нормализацию рекомендуется проводить по выражению, позволяющему каждый вектор-столбец матрицы $\bar{P}$ (см. табл.) делить на ее норму:

$$
\bar{x}_{i j}=\frac{x_{i j}}{\sqrt{\sum_{i=1}^{m} x_{i j}^{2}}} ; \quad \forall i j ; \quad i=\overline{1, m} ; \quad j=\overline{1, n} .
$$

В этом случае каждый вектор-столбец нормализованной матрицы $\bar{P}$ имеет одну и ту же длину. Полученные по выражению (1) числа не имеют дименсий, поэтому можно сравнивать нормализованные значения различных показателей.

В результате нормализации получается матрица принятия решений $\bar{P}$ :

$$
\bar{P}=\begin{gathered}
B_{1} \\
B_{2} \\
\vdots \\
B_{i} \\
\vdots \\
B_{m}
\end{gathered}\left[\begin{array}{cccccc}
r_{11} & r_{12} & \ldots & r_{1 j} & \ldots & r_{1 n} \\
r_{21} & r_{22} & \ldots & r_{2 j} & \ldots & r_{2 n} \\
\vdots & \vdots & \vdots: \vdots & \vdots & : \vdots \vdots & \vdots \\
r_{i 1} & r_{i 2} & \ldots & r_{i j} & \ldots & r_{i n} \\
\vdots & \vdots & \vdots: \vdots & \vdots & \vdots: \vdots & \vdots \\
r_{m 1} & r_{m 2} & \ldots & r_{m j} & \ldots & r_{m n}
\end{array}\right] .
$$


Этап 3. Определение взвешенной нормализованной матрицы принятия решений (МПР). Проводится умножение матрицы $\bar{P}$ на вектор значений весомости ЧКО.

$$
q=\left(q_{1}, q_{2}, \ldots, q_{j}, \ldots, q_{n}\right), \quad \forall j=\overline{1, n} ;
$$

В результате умножения определяется матрица принятия решений $\hat{P}$

$$
\hat{P}=\bar{P} \times q=\left[r_{i j}\right] \cdot\left[q_{j}\right]=\underset{B_{2}}{B_{1}}\left[\begin{array}{cccccc}
f_{11} & f_{12} & \ldots & f_{1 j} & \ldots & f_{1 n} \\
B_{i} & \vdots \\
f_{21} & f_{22} & \ldots & f_{2 j} & \ldots & f_{2 n} \\
\vdots & \vdots & \vdots: \vdots & \vdots & : \vdots: & \vdots \\
f_{m} & f_{i 2} & \ldots & f_{i j} & \ldots & f_{i n} \\
\vdots & \vdots & \vdots:: & \vdots & : \vdots \vdots & \vdots \\
f_{m 1} & f_{m 2} & \ldots & f_{m j} & \ldots & f_{m n}
\end{array}\right] .
$$

Этап 4. Определение идеального и негативно-идеального варианта. Определение идеального варианта осушествляется по выражению (5):

$$
\begin{aligned}
B^{+} & =\left\{\left[\left(\max _{i} f_{i j} / j \in J\right),\left(\min _{i} f_{i j} / j \in J^{I}\right)\right] / i=\overline{1, m}\right\}= \\
& =\left\{f_{1}^{+}, f_{2}^{+}, \ldots, f_{n}^{+}\right\},
\end{aligned}
$$

где $J$ - множество индексов показателей, для которых большие значения являются лучшими (максимизируемые, мажорируемые ПЭ);

$J^{I}$ - множество показателей, меньшие значения которых лучше (минимизируемые, минорируемые ПЭ).

Определение негативно-идеального варианта осуществляется по выражению (6):

$$
\begin{aligned}
B^{-} & =\left\{\left[\left(\min _{i} f_{i j} / j \in J\right),\left(\max _{i} f_{i j} / j \in J^{I}\right)\right] / i=\overline{1, m}\right\}= \\
& =\left\{f_{1}^{-}, f_{2}^{-}, \ldots, f_{n}^{-}\right\} .
\end{aligned}
$$

Таким образом, вариант $B^{+}$соответствует наиболее предпочтительному, вариант $B^{-}-$ наименее предпочтительному

Этап 5. Определение меры разделенности (расстояния) между вариантами. Расстояние между каждым вариантом возможного строительного решения и идеальным определяется по выражению (7):

$$
L_{i}^{+}=\sqrt{\sum_{j=1}^{n}\left(f_{i j}-f_{j}^{+}\right)^{2}}, \quad \forall i, \quad i=\overline{1, m} .
$$

Расстояние между $i-$ м и негативно-идеальным вариантом определяется по выражению (8): 


$$
L_{i}^{-}=\sqrt{\sum_{j=1}^{n}\left(f_{i j}-f_{j}^{-}\right)^{2}}, \quad \forall i, \quad i=\overline{1, m}
$$

Этап 6. Определение относительной близости сравниваемых вариантов строительных решений идеальному варианту $\mathrm{K}_{\text {Бит }} \mathrm{K}_{\text {Бит }}$ определяется по выражению (9):

$$
K_{\text {Бит } i}=\frac{L_{i}^{-}}{L_{i}^{+}+L_{i}^{-}}, \quad \forall i, \quad i=\overline{1, m} .
$$

Из выражения (9) нетрудно видеть, что

$0 \leq K_{\text {Бит } i} \leq 1$, при этом

$$
K_{\text {Бит } i}=\left\{\begin{array}{lll}
1, & \text { если } & B_{i}=B^{+}, \\
0, & \text { если } & B_{i}=B^{-} .
\end{array}\right.
$$

Чем ближе $\mathrm{K}_{\text {Бит }} \mathrm{k} 1$, тем ближе вариант $B_{i} \mathrm{k} B^{+}$. Поэтому после выполнения расчетов по приведенному алгоритму нетрудно определить предпочтительность вариантов строительных решений, то есть лучшим является тот вариант, для которого величина $\mathrm{K}_{\text {Бит }}$ является наибольшей.

Этап 7. Определение предпочтительности вариантов строительных решений. На основании результатов расчета по выражению (9), то есть $\mathrm{K}_{\text {бит }}$ строится ряд предпочтительности применения вариантов возможных строительных решений, например:

$$
\left\{\vec{B}_{i}\right\}=\left\{B_{1} \succ B \succ \ldots \succ B_{i} \succ B_{m}\right\}, \quad \forall i=\overline{1, m}
$$

Этап 8. Принятие базовых ЧКО. Базовые значения ЧКО принимаются на основании этих значений варианта, выбранного в качестве базового варианта строительного решения:

$$
f_{\mathrm{Б}}=\left(f_{\mathrm{B} 1}, f_{\mathrm{Б} 2}, \ldots, f_{\mathrm{Б} j}, \ldots, f_{\mathrm{Б} n}\right), \quad \forall i j, \quad i=\overline{1, m} \quad j=\overline{1, n} .
$$

Этап 9. Сравнение базовых ЧКО с утвержденными прогрессивными удельными показателями (УПУП). Если известны значения УПУП, осуществляется проверка удовлетворения ЧКО значениям УПУП:

$$
\begin{aligned}
& f_{\mathrm{B} j}>b_{j}, \\
& f_{\mathrm{B} j}<b_{j},
\end{aligned}
$$

где $b=\left(b_{1}, b_{2}, \ldots, b_{j}, \ldots, b_{n}\right)$ - значения УПУП $(j=\overline{1, n})$.

Если некоторые значения ЧКО хуже значений УПУП, в качестве базовых величин принимаются значения УПУП.

Полученный на этапе 7 ряд предпочтительности применения вариантов строительных решений может быть использован как для выбора наиболее рациональных вариантов для непосредственного практического применения, так и для целей прогнозирования более прогрессивных строительных решений. 
Если качественные характеристики по каким-либо причинам не могут быть квантифицированы, задачи определения предпочтительности и выбора базового проектного решения решаются с использованием метода упорядочения возможных вариантов решений $[8,11]$ или осушествления выбора подмножества недоминируемых вариантов $[8,9,10] \mathrm{c}$ последующим выбором специалистом, принимающим решение (СПР), единственного варианта.

Принятие научно обоснованных ТЭП и $\mathrm{KX}$ способствует повышению уровня проектных решений и разработке проектов с учетом новейших достижений техники и технологии строительного производства.

\section{3. Оценка качества принимаемых проектных решений}

Оценка качества принимаемого проектного решения может быть выполнена путем его сравнения с базовым решением. ЧКО базового и полученного проектного решения записываются в виде исходной матрицы принятия решения:

$$
P=B_{\bar{n}} B_{\overline{\mathrm{b}}}\left[\begin{array}{cccccc}
x_{\mathrm{b} 1}, & x_{\overline{\mathrm{L}} 2}, & \ldots, & x_{\mathrm{b} j}, & \ldots, & x_{\mathrm{L} n} \\
x_{\bar{n} 1}, & x_{\bar{n} 2}, & \ldots, & x_{\bar{n} j}, & \ldots, & x_{\bar{n} n}
\end{array}\right],
$$

где $B_{\mathrm{Б}}$ и $B_{\bar{n}}-$ соответственно базовый и полученный варианты.

Используя значения ЧКО, приведенные в матрице $P$ (14), по выражению (1) матрица $P$ нормализуется в матрицу $\bar{P}$ :

$$
\bar{P}={ }_{B_{\overline{\mathrm{b}}}}^{B_{\bar{n}}}\left[\begin{array}{llllll}
\bar{x}_{\mathrm{b} 1}, & \bar{x}_{\mathrm{B} 2}, & \ldots, & \bar{x}_{\mathrm{B} j}, & \ldots, & \bar{x}_{\overline{\mathrm{b}} n} \\
\bar{x}_{\bar{n} 1}, & \bar{x}_{\bar{n} 2}, & \ldots, & \bar{x}_{\bar{n} j}, & \ldots, & \bar{x}_{\bar{n} n}
\end{array}\right] .
$$

Если известны исходные коэффициенты весомости ЧКО, матрица $\bar{P}$ (15) преобразуется в матрицу $\hat{P}$ по выражению (4).

Лучший вариант определяется по критерию средневзвешенного успеха принимаемого множества (СУПР 1) по выражению [1]:

$$
K_{2}=B_{i}=\left\{B_{i} / B_{i} \in B_{i} \bigcap \max _{i} \frac{1}{n} \sum_{j=1}^{n} q_{j} \bar{x}_{i j} \bigcap \max _{i} \sum_{j=1}^{n} q_{j} \bar{x}_{i j}\right\}, \quad \forall i j, \quad i=\overline{1, m} ; \quad j=\overline{1, n}
$$

После перехода на принятые в статье обозначения:

$$
\begin{aligned}
& K_{\mathrm{Б}}=\sum_{j=1}^{n} \bar{x}_{\mathrm{B} j} q_{j} \quad \text { и } \\
& K_{\bar{n}}=\sum_{j=1}^{n} \bar{x}_{\bar{n}_{j}} q_{j} .
\end{aligned}
$$


где $\bar{x}_{\bar{b} j}$ и $\bar{x}_{\bar{n} j}$ - значения $j$-го ЧКО соответственно для базового и принятого вариантов проекта.

Если веса значимости ЧКО отсутствуют, лучший вариант определяется по критерию успеха принимаемого решения УПР 2 [1], то есть

$$
K_{3}=B_{i}=\left\{B_{i} / B_{i} \in B_{i} \bigcap \max _{i} \prod_{j=1}^{n} \bar{x}_{i j}\right\}, \quad \forall i j, \quad i=\overline{1, m} ; \quad j=\overline{1, n} .
$$

После перехода на принятые в статье обозначения

$$
\begin{aligned}
& K_{\mathrm{b}}=\prod_{j=1}^{n} \bar{x}_{\mathrm{b} j} . \\
& K_{\bar{n}}=\prod_{j=1}^{n} \bar{x}_{n j} .
\end{aligned}
$$

Если $K_{\mathrm{b}} \leq K_{\bar{n}}$, то полученное проектное решение не уступает базовому.

\section{4. Заключение}

Предложенный метод удобен для пользования. При незначительном количестве вариантов и характеризуюших их полидименсиональных похазателей эффективности расчеты несложны и их можно успешно выполнить без персонального компьютера. При большем количестве оцениваемых вариантов или при использовании разработанного метода системотехнической оценки и определения предпочтительности проектных решений в составе экспертных систем необходимо использование компьютера. Предлохенный метод отличается простотой и наглядностью, поэтому он может быть использован и в учебном процессе при работе со студентами строительных специальностей.

\section{Јитература}

1. Э.К.Завадскас. Системотехническая оценка технологических решений строительного производства. Ленинград: Стройиздат, 1991. 257 с.

2. Э.К.Завадскас. Многоцелевая селектоновация технологических решений строительного производства (теоретические основы). Вильнюс, 1989. 107 с.

3. E.K.Zavadskas. Mehrzielselektierung bei der Bauvorbereitung // Statyba, No 3(3), Vilnius: Technika, 1995, p. 58-80.

4. E.T.Jaynes. Information Theory and Statistical Mechanics // Physical Review. Vol. 104, No 4,1957 , p. 620-630.

5. Э.К.Завадскас. Комплексная оценка и выбор ресурсосберегаюших решений в строительстве. Вильнюс: Мокслас, 1987. 212 с. 
6. E.Zavadskas, A.Kaklauskas. Automated multivariant design of buildings, multipurpose comprehensive evaluation and selection of the most efficient versions. Aalborg: Aalborg Universitetscenter, 1991. $65 \mathrm{p}$.

7. E.Zavadskas, A.Kaklauskas. Multiple criteria analysis of projects. Aalborg: Aalborg University, 1992. $93 \mathrm{p}$.

8. E.Zavadskas, F.Peldschus, A.Kaklauskas. Multiple criteria evaluation of projects in construction. Vilnius: Technika, 1994. 226 p.

9. Б.Руа. Классификация и выбор при наличии нескольких критериев (метод Электра): Пер. с англ. // Вопросы анализа и процедуры принятия решений. Москва: Мир, 1976, c. $80-107$.

10. B.Roy. Partial Preference Analysis and Decision-Aids: The Fuzzy Outranking Relation Concept // Conflikting objectives in decisions. Wiley New York: Wiley, 1977, p. 40-75.

11. J.Paelink. Qualitative Multiple - Criteria Analysis, Environmental Protection and Multiregional Development // Netherlands Economic Institute: Foundations of Empirical Economic Research, 1975, No 10, 27 p.

\section{APIBENDRINTAS PROJEKTINIU SPRENDIMU STATYBOJE SISTEMOTECHNINIS IVERTINIMAS IR NAUDOJIMO PRIORITETU NUSTATYMAS}

\section{E.K.Zavadskas}

S a n $t r a u k$ a

Nagrinejama palyginimo bazes problema, iškylanti atliekant projektinių sprendimy variantu sistemotechnini jvertinimą. Pasiūlyta nauja metodika, algoritmas ir programa, leidžiantys bazinio projektinio sprendimo varianto parinkime dalyvauti ir užsakovui, kuris turi galimybę kompiuterio pagalba dialogo režime išreikšti savo preferencijas, pasirenkant projektini sprendimą. Pasiūlytas apibendrintas projekto naudingumo kriterijus, kuris skaičiuojamas pagal nuotolius nuo vadinamojo idealaus projektinio varianto, sudaryto iš visu vertinamy variantų geriausių efektyumo rodikliu. Sistemotechninio jvertinimo rezultatas - varianty naudojimo prioritety eilutè.

\section{A GENERALIZED SYSTEMOTECHNICAL EVALUATION AND DETERMINATION OF THE PRIORITIES IN BUILDING DESIGN DECISIONS}

\section{E.K.Zavadskas}

S u m m a r y

The paper discusses the problem of determination of efficiency indices used in comparative evaluation of building design dicesions. The methodology, an algorithm and a computing programme for determining basic technical and economical indices and qualitative characteristics have been worked out. A method for determining the most preferable variant of design decisions based on the criterion of utility has been suggested in the article. The result of the systemotechnical evaluation is a line of application priorities. 